\title{
The model developed for stress-induced structural phase transformations of micro-crystalline silicon films
}

\author{
Chang-Fu Han ${ }^{1}$ and Jen-Fin Lin'1,2*
}

The nanoindentations were applied to island-shaped regions with metal-induced Si crystallizations. The experimental stress-strain relationship is obtained from the load-depth profile in order to investigate the critical stresses arising at various phase transitions. The stress and strain values at various indentation depths are applied to determine the Gibbs free energy at various phases. The intersections of the Gibbs free energy lines are used to determine the possible paths of phase transitions arising at various indentation depths. All the critical contact stresses corresponding to the various phase transitions at four annealing temperatures were found to be consistent with the experimental results.

Keywords: Silicon films; Phase transitions; Stress-strain model

Citation: Chang-Fu Han and Jen-Fin Lin, "The model developed for stress-induced structural phase transformations of micro-crystalline silicon films", Nano-Micro Lett. 2, 68-73 (2010). doi: 10.5101/nml.v2i2.p68-73

The depth-sensing indentation (nanoindentation) test is a powerful tool for determining the mechanical properties of bulk specimens [1-6] and thin film/substrate systems [7-9]. Atomistic simulations of nanoindentation have been performed to investigate anisotropic effects in elastic and incipient plastic behavior under nanoindentation [10-12]. The determination of atomistic fracture modes under various loading conditions is essential to understanding nanomechanics. Pan et al. [13] reported first-principles calculations that show intriguing indenter-angle-sensitive fracture modes and stress responses at the incipient plasticity of strong covalent solids. A basic micromechanical model for the deformation of solids with only one tuning parameter has been introduced. The model can reproduce observed stress-strain curves, acoustic emissions and related power spectra, event statistics, and geometrical properties of slip, with a continuous phase transition from brittle to ductile behavior [14-16]. Nanometer scale indents have been written in a cross-linked polystyrene sample, and their relaxation has been studied at annealing temperatures well below the glass transition of the polymer [17]. The indentation stress-strain curve exhibits a series of yielding events, attributed to the nucleation and movement of dislocations [18]. In porous silicon [19], a transition from the diamond phase to a high density amorphous (HDA) phase has been reported. Nanocrystalline silicon [20] undergoes a direct transition from diamond to a simple hexagonal structure. One such prediction was a kinetically hindered first order amorphous to amorphous phase transition in $\mathrm{SiO}_{2}$ [21]. An experiment has recently confirmed such a first order amorphous to amorphous phase transition [22]. Such calculations require candidate structures which may be obtained from constant pressure simulations and sometimes experiments $[23,24]$. In the present study, a theoretical model is developed

1Department of Mechanical Engineering

${ }^{2}$ Center for Micro/Nano Science and Technology National Cheng Kung University, Tainan 701, Taiwan, R.O.C.

*Corresponding author. Email: jflin@mail.ncku.edu.tw 
using contact stress-strain analysis and Gibbs free energy to investigate the stress-induced phase transitions arising at various nanoindentation depths of microcrystalline silicon $(\mu \mathrm{c}-\mathrm{Si})$. Four kinds of $\mathrm{Al} / \mathrm{a}-\mathrm{Si} / \mathrm{Glass} 7740$ specimen with silicon crystallizations were prepared at four annealing temperatures $\left(350^{\circ} \mathrm{C}, 400^{\circ} \mathrm{C}, 450^{\circ} \mathrm{C}\right.$, and $\left.500^{\circ} \mathrm{C}\right)$, respectively. The $\mathrm{Al}$ film was then removed by wet etching. A model with originality is developed in order to transform the experimental load-depth results into stress-strain relationships. The stress-strain results allow us to calculate the values of Gibbs free energy for the possible phases arising at various indentation depths. The intersection points of these Gibbs free energy lines can be used to determine the possible paths of phase transitions.

\section{Stress Analyses of Composite Film}

During the indentation test, the composite film layer may delaminate from its substrate if the indentation depth, thus the indentation load, is sufficiently high. The deformation of the composite film under a uniform pressure distribution can thus be imagined as the deflection of a membrane. From the bending assuming elastic deformation, the internal stress $\sigma_{I}$ created in the undeformed composite film with a thickness of $t^{*}$. The internal stress $\sigma_{I}$ and the contact force Fc satisfy the following relation [25-26]:

$\sigma_{I}=\frac{F_{c}}{12 \sqrt{3} \cdot h_{c} \cdot t^{*}}$

The values of $\mathrm{Fc}$ and hc can be obtained from the $\operatorname{load}(\mathrm{P})-\operatorname{depth}(\mathrm{h})$ analyses of the nanoindentation test [26,27]. The contact depth, hc, can be obtained using the method of Oliver and Pharr [2]. Recent experiments have revealed that the results of nanoindentation strongly depend on indenter geometry [28-30]. Here, we examine the atomistic response to nanoindentation by a sharp indenter with various centerlineto-face angles $\alpha$. From the stress response at incipient plasticity developed by Pan et al. [13], the contact stress $\sigma_{c}$ and indentation

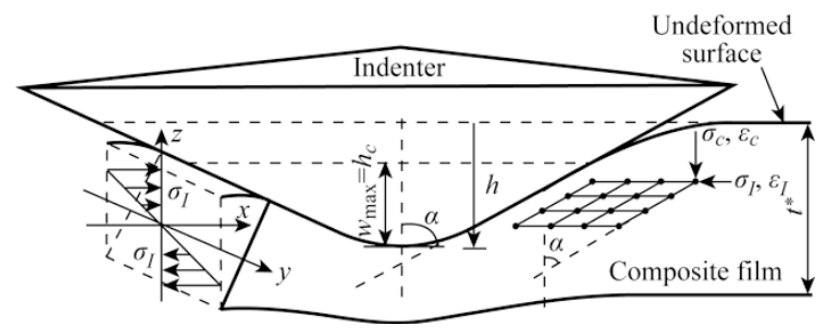

FIG. 1. Scheme that demonstrates how the indentation depth of the composite film under uniform pressures is related to the internal stress $\sigma_{I}$ and contact stress $\sigma_{c .}$ strain $\varepsilon_{c}$ in Fig. 1 are related by:

$\frac{\sigma_{c}}{\sigma_{I}}=\frac{\varepsilon_{c}}{\varepsilon_{I}}=\tan \alpha$

$\sigma_{c}$ can be obtained from the above equation since the angle $\alpha$ is known. The application of contact stress to solids leads to a diverse collection of interesting phenomena. The most dramatic behavior may be the stress-induced phase transitions. Stress-induced phase transitions in microcrystalline silicon ( $\mu \mathrm{c}-\mathrm{Si}$ ) have been extensively studied [19-24]. In order to study the model of crystallization, the volume-dependent Gibbs free energy is evaluated. To calculate the static structural properties of the silicon phase, the total energy $E_{\text {tot }}$ can be obtained in a polynomial form, expressed as Murnaghan's equation of state [31]:

$$
E_{t o t}(V)=\frac{B_{0} V}{B^{\prime}\left(B^{\prime}-1\right)} \cdot\left[B^{\prime}\left[1-\frac{V_{\min }}{V}\right]+\left[\frac{V_{\min }}{V}\right]^{B^{\prime}}-1\right]+E_{\text {min }}
$$

and

$V_{t}=\frac{V}{V_{\min }}$

where $V$ and $V_{t}$ are the volume and transition volume per atom at various phases of silicon, respectively. $V_{\min }$ is the volume per atom corresponding to the minimum total energy $E_{\min }$ per atom. The crystal data at diamond-Si, body-centered tetragonal $(\beta$-tin $\mathrm{Si})$, primitive hexagonal ( $p h$-Si), and HDA phases of silicon includes $V_{t}($ diamond-Si $)=0.89, \quad V_{t}(\beta$-tin $\mathrm{Si})=0.71, \quad V_{t}(p h-\mathrm{Si})=0.69, \quad V_{t}(\mathrm{HDA})=0.65, \quad V_{\min }($ diamond $-\mathrm{Si})=$ $20.76 \AA^{3}, \quad V_{\min }(\beta$-tin $\mathrm{Si})=15.42 \AA^{3}, \quad V_{\min }(p h-\mathrm{Si})=15.66 \AA^{3}$, $V_{\min }(\mathrm{HDA})=15.87 \AA^{3}, E_{\min }$ (diamond-Si) $=-108.00 \mathrm{eV}, E_{\min }(\beta$-tin $\mathrm{Si})=-107.71 \mathrm{eV}, \quad E_{\min }(p h-\mathrm{Si})=-107.68 \mathrm{eV}$, and $E_{\min }(\mathrm{HDA})=$ $-107.53 \mathrm{eV}$, as obtained from the studies in [23,24,31]. In the present study, $B_{0}$ and $B^{\prime}$ are the bulk modulus and the pressure derivative of the bulk modulus for silicon with $V_{\min }$, respectively. This study calculates the indentation stress based on the thermodynamics theorem. When a transition occurs, the Gibbs free energy at zero temperature between the two phases is the same. The Gibbs free energy is written as:

$G=E_{t o t}+\sigma_{c} V$

The contact stresses corresponding to these phase transitions can be evaluated from Eq. (5) if the Gibbs free energy $(G)$ and the total energy $\left(E_{\text {tot }}(V)\right.$ in Eq. (3)) are available. These stresses predicted by the proposed model are compared with the experimental results in order to validate the proposed model for the prediction of phase transition.

http://www.nmletters.org 


\section{Results and Discussions}

The indentation tests were carried out on a Nano Indenter XP (MTS, USA) tester. All experiments were carried out using the Berkovich indenter, which was made of diamond (Young's modulus $E=1140 \mathrm{GPa}$, Poisson's ratio $v=0.07$ ). In this study, the substrates used for the aluminium (Al)/amorphous silicon (a-Si)-layer structure were made of Corning glass (code 7740). a-Si films with a thickness of $200 \mathrm{~nm}$ were deposited onto the glass substrate using an ultra-high vacuum ion beam sputtering system (UHV-IBS, model IBS-2000 ULVAC, Japan) with a high purity target of $\mathrm{Si}(99.999 \%)$. An Al layer with a thickness of $50 \mathrm{~nm}$ was thermally evaporated and deposited onto the a-Si layer. When the aluminum depositions were finished, the glass was cut into small pieces and annealed at temperatures of $350^{\circ} \mathrm{C}$, $400^{\circ} \mathrm{C}, 450^{\circ} \mathrm{C}$, and $500^{\circ} \mathrm{C}$ respectively. The remaining $\mathrm{Al}$ was then removed by wet selective etching after the annealing process. The microstructure was characterized using focused ion beam microscopy (FIB, FEI Nova-200 NanoLab compatible, USA) and high-resolution transmission electron microscope (HR-TEM, JEM-2100F, JEOL, Japan) was used to investigate the density of Si crystallization. In the present study, the Al (50 $\mathrm{nm}$ )/a-Si (200 nm)/Glass 7740 (substrate) specimens were treated at four annealing temperatures and at room temperature $\left(25^{\circ} \mathrm{C}\right)$. No silicon crystallization was found at the a-Si layer for the specimen that was prepared without further annealing (at room temperature), as shown in Fig. 2(a). However, $\mathrm{Si}$ crystallizations appear as grains with various sizes at the a-Si layer for annealing temperatures $(T)_{\text {annealing }} \geqslant 350{ }^{\circ} \mathrm{C}$. This specimen was annealed at a temperature of $350^{\circ} \mathrm{C}$ as shown in Fig. 2(b). In this figure, many black island-shaped stains can be seen on the annealed Si layer.

Figure 3(a) shows the cross section of the nanoindentation applied at a stain in Fig. 2(b) with silicon crystallizations. The white dashed lines enclose the final indentation cavity. There is a dark band surrounding the cavity. Point B in Fig. 3(b) is exactly beneath the cavity tip. Point A is considerably far from the boundary of the indentation cavity, but is still in the region of Si crystallization. Point $\mathrm{C}$ is exactly beneath the cavity tip and near the interface of the $(\mathrm{a}-\mathrm{Si}+\mu \mathrm{c}-\mathrm{Si})$ film layer and the Glass 7740 substrate, but still at the (a-Si+ $\mu \mathrm{c}-\mathrm{Si})$ film layer. Point D is located beneath point $\mathrm{C}$; it is at the protrusion of the Si-layer into the region originally belonging to the Glass 7740 substrate. The TEM diffraction pattern in the bottom-left corner shows point A being induced to the primitive hexagonal silicon phase by the contact stress $\left(\sigma_{c}\right)$. The TEM diffraction pattern for point $\mathrm{B}$ is shown in the bottom-right corner of Fig. 3(c); it was identified as the HDA Si phase [20]. The TEM diffraction pattern of the phases shown in Fig. 3(d) for point $C$ and point $D$ were identified as largely the amorphous phase, even though very little crystalline behavior is still existent. Therefore point $\mathrm{C}$ and point $\mathrm{D}$ are located in the region affected by the stress-induced crystallizations. The coexistence of the amorphous and crystalline regions in the (a-Si+ $\mu \mathrm{c}-\mathrm{Si})$ film layer near the indentation cavity confirms the validity of the phase transitions predicted by the proposed model.

Figure 4(a) shows the $\sigma_{c}-\varepsilon_{c}$ curve of the Al/a-Si/Glass 7740 specimen annealed at $350{ }^{\circ} \mathrm{C}$ after nanoindentation obtained from the proposed model. The non-smooth curve indicates that stress-induced phase transitions occurred at various critical stresses $\left(\sigma_{\mathrm{c} \_i}, i=1,2,3 \cdots\right)$. The possible phases are identified as diamond cubic $\mathrm{Si}$, the body-centered tetragonal ( $\beta$-tin $\mathrm{Si}$ ), the primitive hexagonal $\mathrm{Si}(p h-\mathrm{Si})$, and HDA Si phases [23,24,31]. Figure 4(b) shows the four lines of Gibbs free energy corresponding to the four possible phases of silicon as a function of $\sigma_{c}$. As Fig. 4(b) shows, the specimen begins with the diamond Si phase; the $G_{\text {diamond-Si }}$ line intersects the $G_{\beta \text {-tin }}$ Si line at the point where $\sigma_{c}=6.04 \mathrm{GPa}$. The $\sigma_{c}$ value is defined as the dashed line of $\sigma_{c \_2}$ in Fig. 4(a). After this point, an increase in the indentation depth, and thus the $\sigma_{c}$ value, allows the $\mathrm{Si}$ layer to operate in the $\beta$-tin Si phase, which is indicated by the arrow on the $G_{\beta-\text { tin Si }}$ line. When the $\sigma_{c}$ value increases to $8.65 \mathrm{GPa}$, the $G_{\beta \text {-tin Si }}$ line intersects the $G_{p h \text {-Si }}$ line. Therefore, the $\beta$-tin Si phase transforms to the primitive hexagonal phase (ph-Si) if the $\sigma_{c}$ value is higher than $8.65 \mathrm{GPa}$. This value is here defined as $\sigma_{c \_3}$. As Fig. 4(b) shows, the diamond Si phase can also directly transform into the HDA Si phase. The critical contact stress corresponding to this direct transition is $8.82 \mathrm{GPa}$. Figure 4(c) shows the Raman spectra for the specimens annealed at $350{ }^{\circ} \mathrm{C}$ before and after nanoindentations, respectively. In the specimen before nanoindentation, the sole intensity peak appears at $518 \mathrm{~cm}^{-1}$. This peak in the Raman band is identified as the diamond cubic (Si-I) phase. For the Raman bands in the cavity after nanoindentation, peaks at 518 $\mathrm{cm}^{-1}, 476 \mathrm{~cm}^{-1}, 446 \mathrm{~cm}^{-1}, 403 \mathrm{~cm}^{-1}$, and $357 \mathrm{~cm}^{-1}$ were observed in the specimen. The Raman bands at $357 \mathrm{~cm}^{-1}$ and $446 \mathrm{~cm}^{-1}$ were identified as the rhombohedra (r8) Si-XII phase and the body-center-cubic (bc8) Si-III phase, respectively [29,32-35]. These two phases appeared in the unloading process of nanoindentation only. The peaks corresponding to $403 \mathrm{~cm}^{-1}$, $476 \mathrm{~cm}^{-1}$, and $518 \mathrm{~cm}^{-1}$ are the three phases in the loading process. The peak at $403 \mathrm{~cm}^{-1}$ was identified as the body-centered tetragonal $(\beta$-tin $\mathrm{Si}$ ) $\mathrm{Si}$-II and primitive 


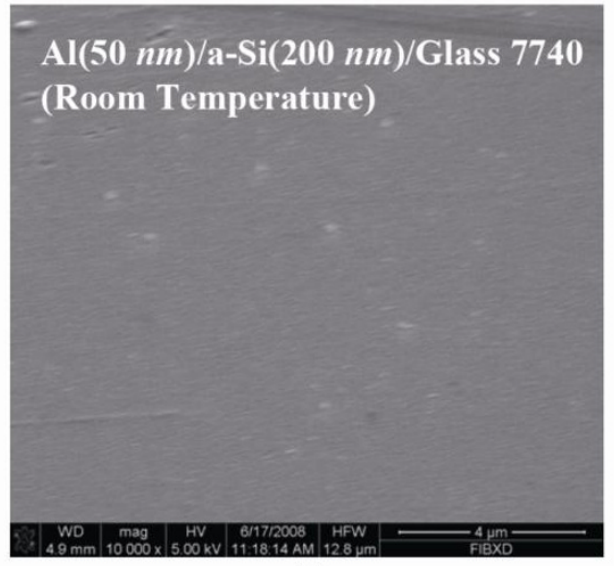

(a)

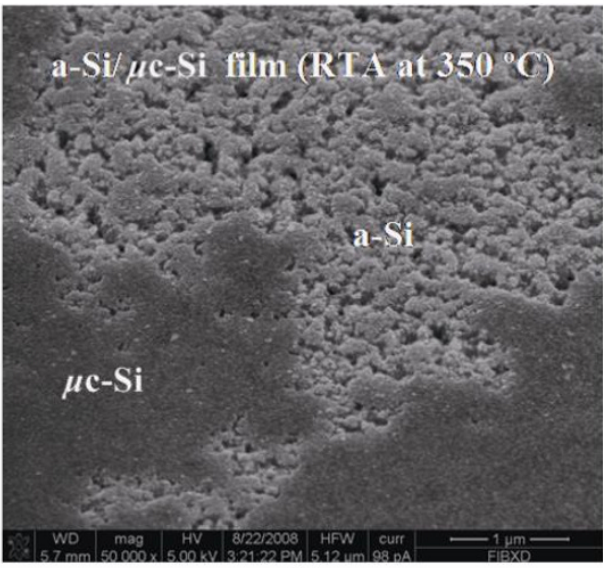

(b)

FIG. 2. Surface image of the $\mathrm{Al}(50 \mathrm{~nm}) / \mathrm{a}-\mathrm{Si}(200 \mathrm{~nm}) / \mathrm{Glass} 7740$ specimen at room temperature $\left(25^{\circ} \mathrm{C}\right)$. (b) Morphology of the $\mathrm{Si}$ layer after etching of the $\mathrm{Al}$ layer at an annealing temperature of $350^{\circ} \mathrm{C}$.

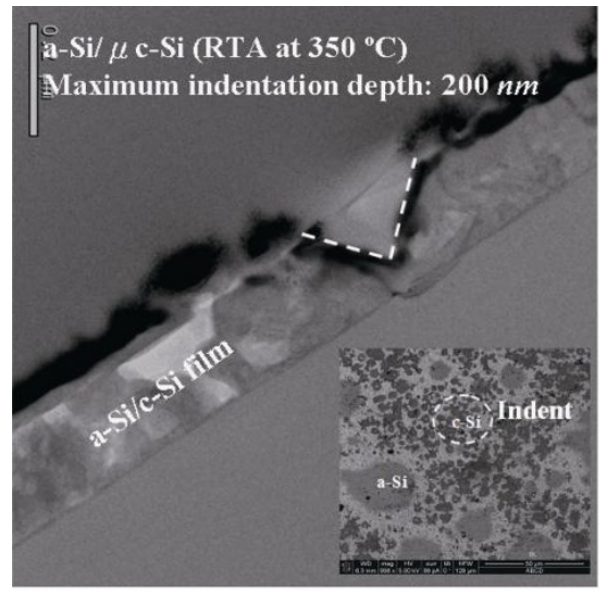

(a)

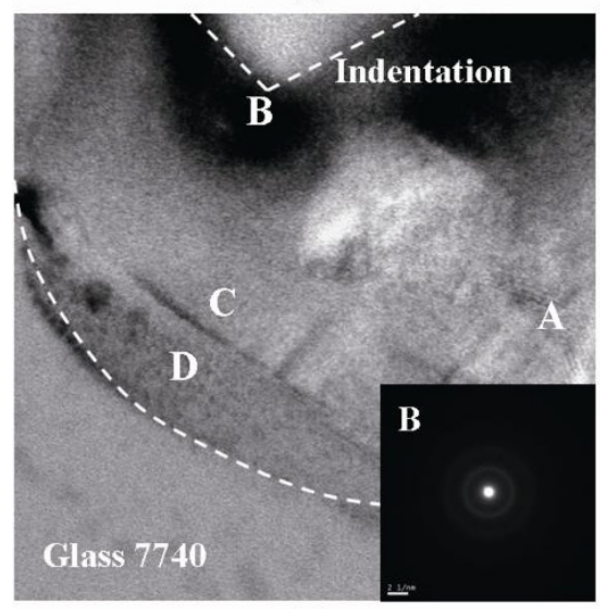

(c)

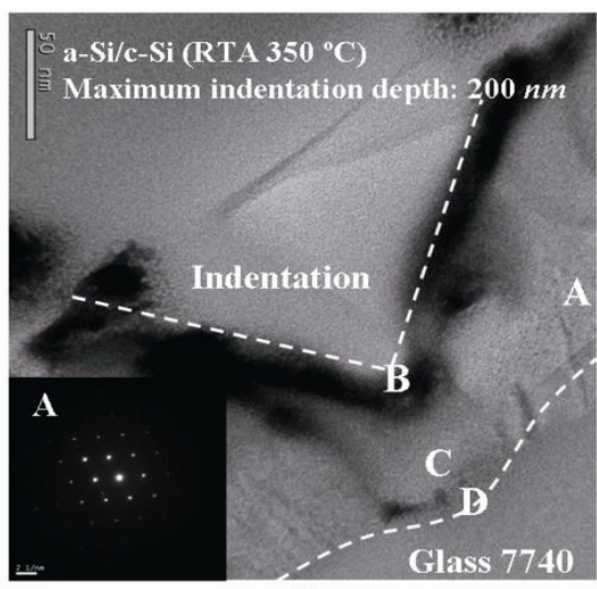

(b)

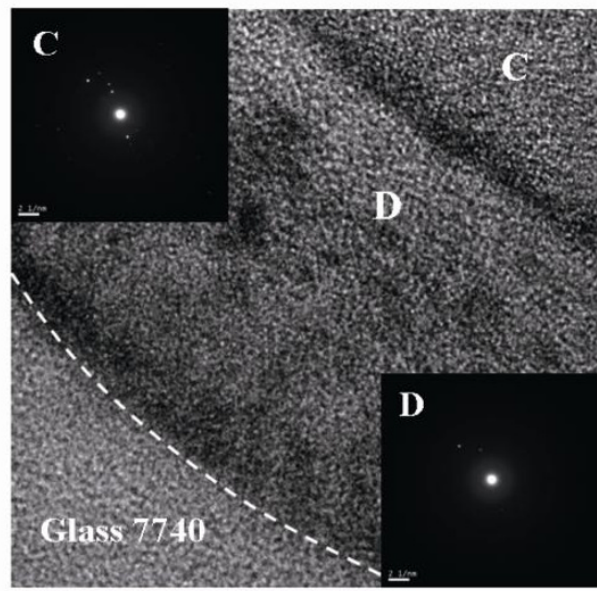

(d)

FIG. 3. Bright field HR-TEM micrographs of (a) the cross-section after indentation, (b) the local magnification of the indentation cavity and the diffraction pattern of point $\mathrm{A}$, (c) the diffraction pattern of point $\mathrm{B}$, and (d) the diffraction pattern of point $\mathrm{C}$ and point $\mathrm{D}$.

hexagonal ( $p h$-Si) Si-V phases [36]. The peak at $476 \mathrm{~cm}^{-1}$ was identified as the amorphous and HDA phases [34]. This phase was created due to the stress-induced phase transition shown at one of the two predicted paths shown in Fig. 4(b). Figure 4(d) shows the experimental $\sigma_{c}-\varepsilon_{c}$ results for the five specimens treated at four annealing temperatures and with no annealing, respectively. The dashed curves denoted by $\sigma_{c_{-} 2}$ and $\sigma_{c_{-3}}$ were obtained from the predictions of the proposed model. These dashed curves intersect the $\sigma_{c}-\varepsilon_{c}$ curves at the points symbolized by " $\mathbf{\Delta}$ ". It was found that the intersection points 


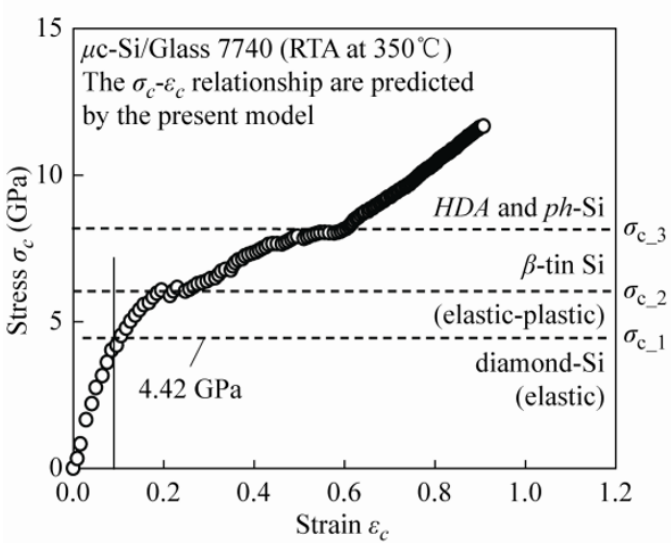

(a)

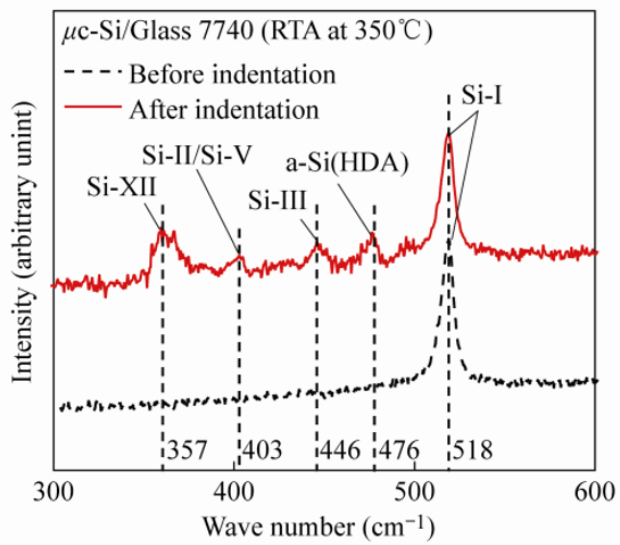

(c)

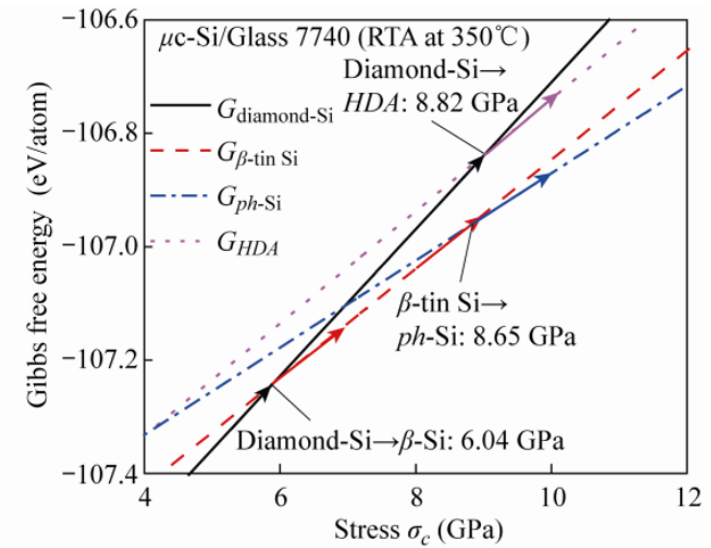

(b)

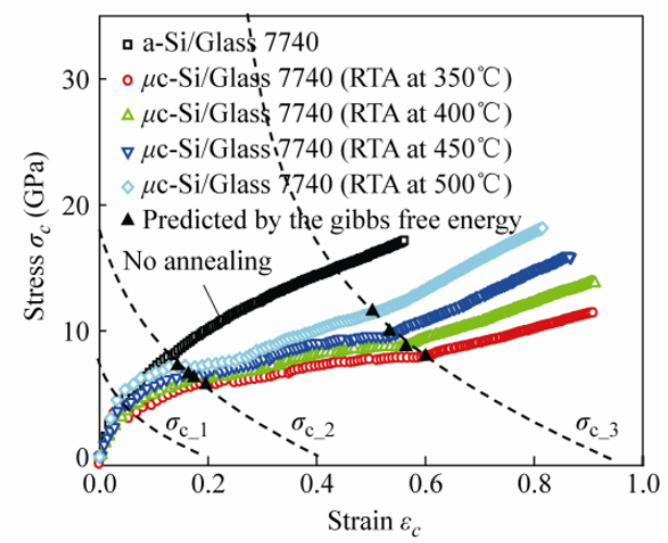

(d)

FIG. 4. (a) Relationship of the contact stress $\sigma_{c}$ and the strain $\varepsilon_{c}$ for the a-Si $/ \mu$ c-Si film annealed at a temperature of $350^{\circ} \mathrm{C}$. (b) Gibbs free energy for the diamond-Si, $\beta$-tin $\mathrm{Si}, p h$-Si, and HDA phases. (c) Raman spectra on the area of $\mu \mathrm{c}-\mathrm{Si}$ at an annealing temperature of $350^{\circ} \mathrm{C}$ before and after indentation. (d) Experimentally obtained the $\sigma_{c_{-} 1}, \sigma_{c_{-} 2}$, and $\sigma_{c_{-} 3}$ curves (dashed) predicted by the proposed model.

are very close to the two turning points of the $\sigma_{c}-\varepsilon_{c}$ curve, respectively, for each of the four curves for annealed specimens. Therefore, the predictions by the proposed model are effective for all the specimens with phase transitions.

\section{Conclusion}

The contact stress $\left(\sigma_{c}\right)$ and contact strain $\left(\varepsilon_{c}\right)$ models developed in the present study for nanoindentations allow us to obtain the experimental $\sigma_{c}-\varepsilon_{c}$ curve and thus identify the critical values for stress-induced phase transitions at various indentation depths. The contact stress and contact strain developed in the proposed model are provided to determine the Gibbs free energy for various phases. The critical contact stresses corresponding to phase transitions can be determined from the intersections of the Gibbs free energy lines. Then, the paths from the start of diamond cubic silicon to the possible final phases corresponding to the maximum indentation depth can be identified. The theoretical predictions of final phases in the present study were confirmed by TEM diffraction patterns and Raman spectra.
This work has been granted by Frontier Materials and Micro/Nano Science and Technology Center, National Cheng Kung University, Taiwan, R.O.C.

Received 10 March 2010; accepted 15 April 2010; published online 22 April 2010.

\section{References}

1. A. A. Volinsky, N. R. Moody and W. W. Gerberich, Acta. Mater. 50, 441 (2002). doi:10.1016/S13596454(01)00354-8

2. W. C. Oliver and G. M. Pharr, J. Mater. Res. 7, 1564 (1992). doi:10.1557/JMR.1992.1564

3. M. Sakai, Acta. Metal. Mater. 41, 1751 (1993). doi:10.1016/0956-7151(93)90194-W

4. S. Suresh and A. E. Giannakopoulos, Scripta Mater. 40, 1191 (1999). doi:10.1016/S1359-6462(99)0001 1-1

5. B. Taljat, T. Zacharia and F. Kosel, Int. J. Solids Struct. 35, 4411 (1998). doi:10.1016/S0020-768 $\underline{3(97) 00249-7}$ 
6. J. S. Field and M. V. Swain, J. Mater. Res. 8, 297 (1993). doi:10.1557/JMR.1993.0297

7. A. C. Fischer-Cripps, Nanoindentation, Springer, New York (2004).

8. T. F. Page, G.M. Pharr, J. C. Hay, W. C. Oliver, B. N. Lucas, E. Herbert and L. Riester, MRS Symp. Proc. 522, 53 (1998).

9. P. J. Wei and J. F. Lin, Surf. Coat. Tech. 200, 2489 (2005). doi:10.1016/j.surfcoat.2004.09.013

10. T. Tsuru and Y. Shibutani, Phys. Rev. B 75, 035415 (2007).doi:10.1103/PhysRevB.75.035415

11. J. K. Mason, A. C. Lund and C. A. Schuh, Phys. Rev. B 73, 054102 (2006). doi:10.1103/PhysRev $\underline{\text { B.73.054102 }}$

12. A. Makagon, M. Kachanov, S. V. Kalinin and E. Karapetian, Phys. Rev. B 76, 064115 (2007). doi:10.1103/PhysRevB.76.064115

13. Z. C. Pan, H. Sun and C. F. Chen, Phys. Rev. B 79, 104102 (2009). doi:10.1103/PhysRevB.79.104102

14. K. A. Dahmen, Y. Ben-Zion and J. T. Uhl, Phys. Rev. Lett. 102, 175501 (2009). doi:10.1103/Phy sRevLett.102.175501

15. C. Kearney, Z. Zhao, B. J. F. Bruet, R. Radovitzky, M. C. Boyce and C. Ortiz, Phys. Rev. Lett. 96, 255505 (2006). doi:10.1103/PhysRevLett.96.25 $\underline{5505}$

16. Y. T. Cheng, W. Y. Ni and C. M. Cheng, Phys. Rev. Lett. 97, 075506 (2006). doi:10.1103/PhysRevLe $\underline{\text { tt.97.075506 }}$

17. A. Knoll, D. Wiesmann, B. Gotsmann and U. Duerig, Phys. Rev. Lett. 102, 117801 (2009). doi:10.1103/PhysRevLett.102.117801

18. M. Lucas, A. M. Leach, M. T. McDowell, S. E. Hunyadi, K.Gall, C. J. Murphy and E. Riedo, Phys. Rev. B 77, 245420 (2008). doi:10.1103/PhysRevB. $\underline{77.245420}$

19. S. K. Deb, M. Wilding, M. Somayazulu and P. F. McMillan, Nature 414, 528 (2001). doi:10.1038/ $\underline{35107036}$

20. S. H. Tolbert, A. B. Herhold, L. E. Brus and A. P. Alivisatos, Phys. Rev. Lett. 76, 4384 (1996). doi:10.1103/PhysRevLett.76.4384
21. D. J. Lacks, Phys. Rev. Lett. 84, 4629 (2000). doi:10.1103/PhysRevLett.84.4629

22. G. D. Mukherjee, S. N. Vaidya and V. Sugandhi, Phys. Rev. Lett. 87, 195501 (2001). doi:10.1103/ PhysRevLett.87.195501

23. M. Durandurdu and D. A. Drabold, Phys. Rev. B 64, 014101 (2000). doi:10.1103/PhysRevB.64. $\underline{014101}$

24. M. Durandurdu and D. A. Drabold, Phys. Rev. B 67, 212101 (2003). doi:10.1103/PhysRevB.67. 212101

25. R. C. Hibbeler, Mechanics of Materials Prentice Hall International, Inc (1997).

26. C. F. Han, B. H. Wu, C. Y. Huang and J. F. Lin, J. Appl. Phys. 105, 023523 (2009). doi:10.1063/ $\underline{1.3056392}$

27. C. F. Han, B. H. Wu, J. F. Lin and C. K. Chung, Nanotechnology 19, 325710 (2008). doi:10.1088/0957-4484/19/32/325710

28. S. R. Kalidindi and S. Pathak, Acta. Mater. 56, 3523 (2008). doi:10.1016/j.actamat.2008.03.036

29. J. Jang, M. J. Lance, S. Wen, T. Y. Tsui, and G. M. Pharr, Acta. Mater. 53, 1759 (2005). doi:10.1016/j. actamat.2004.12.025

30. H. Bei, E. P. George, J. L. Hay and G. M. Pharr, Phys. Rev. Lett. 95, 045501 (2005). doi:10.1103/ PhysRevLett.95.045501

31. F. Zandiehnadem and W. Y. Ching, Phys. Rev. B 41, 162 (1990). doi:10.1103/PhysRevB.41.12162

32. I. Zarudi and L. C. Zhang, Tribology Intern.32, 701 (1999). doi:10.1016/S0301-679X(99)00103-6

33. J. E. Bradby, J. S. Williams and J. Wong-Leung, Appl. Phys. Lett. 77, 3749 (2000). doi:10.1063/ $\underline{1.1332110}$

34. J. E. Bradby, J. S. Williams and J. Wong-Leung, J. Mater. Res. 16, 1500 (2001). doi:10.1557/JMR. $\underline{2001.0209}$

35. I. Zarudi, L. C. Zhang, W. C. D. Cheong and T. X. Yu, Acta. Mater. 53, 4795 (2005). doi:10.1016/j. actamat.2005.06.030

36. H. Olijnyk, Phys. Rev. Lett. 68, 2232 (1992). doi:10.1103/PhysRevLett.68.2232 